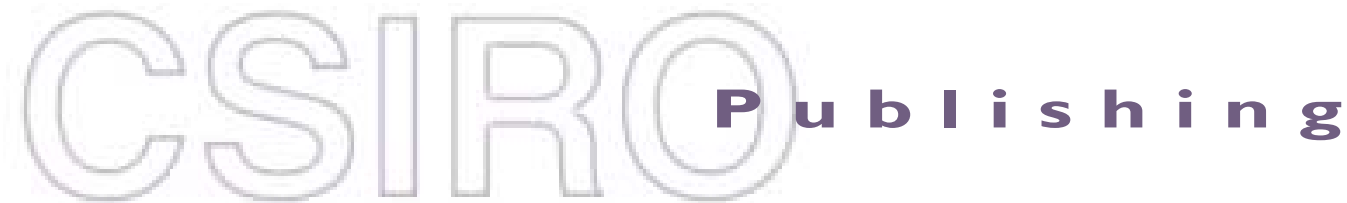

\section{Publications of the Astronomical Society of Australia}

Volume 19, 2002

(C) Astronomical Society of Australia 2002

An international journal of astronomy and astrophysics

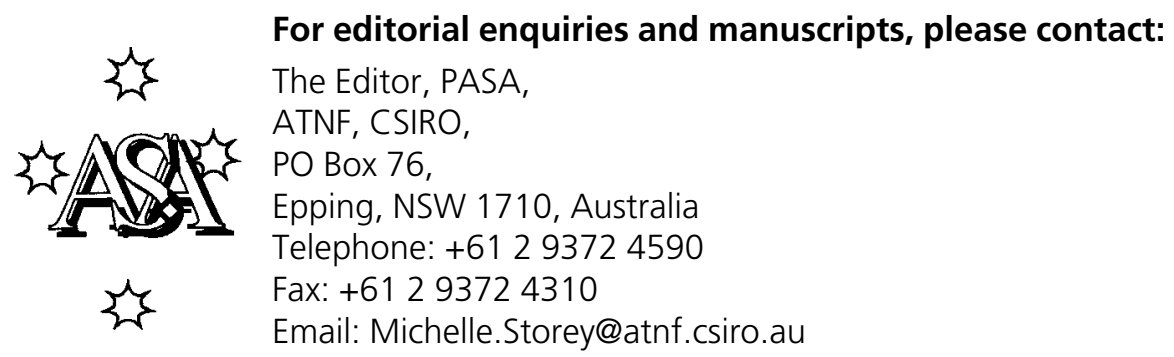

For general enquiries and subscriptions, please contact: CSIRO Publishing PO Box 1139 (150 Oxford St)

Collingwood, Vic. 3066, Australia

Telephone: +6139662 7666

Fax: +61 396627555

Email: publishing.pasa@csiro.au

C S I RO

PUBLISHING Published by CSIRO Publishing

for the Astronomical Society of Australia

www.publish.csiro.au/journals/pasa 


\title{
Microquasars: A Galactic-Extragalactic Connection?
}

\author{
Kinwah $\mathrm{Wu}^{1,2}$, Jason A. Stevens ${ }^{1,3}$ and Diana C. Hannikainen ${ }^{4}$ \\ ${ }^{1}$ Mullard Space Science Laboratory, University College London, \\ Holmbury St Mary, Surrey, RH5 6NT, United Kingdom \\ kw@mssl.ucl.ac.uk \\ ${ }^{2}$ School of Physics, University of Sydney, NSW 2006, Australia \\ ${ }^{3}$ Astronomy Technology Center, Royal Observatory, \\ Blackford Hill, Edinburgh, EH9 3HJ, United Kingdom \\ jas@roe.ac.uk \\ ${ }^{4}$ Department of Physics and Astronomy, Southampton University, \\ Southampton, SO17 1BJ, United Kingdom \\ dch@astro.soton.ac.uk
}

Received 2001 September 24, accepted 2002 February 14

\begin{abstract}
Microquasars are Galactic X-ray binaries which show radio emission from relativistic outflows. Here we argue that the origins of radio emission from microquasars and compact extragalactic radio sources are similar. In particular, both can be explained qualitatively by a model in which shocks are propagating in the relativistic jets. The microquasar GRO J1655-40 is used to illustrate that the spectral evolution of its 1994 radio outburst can be explained by the growth phase of the generalised-shock model, similar to the case of the quasar $3 \mathrm{C} 273$.
\end{abstract}

Keywords: black hole physics — galaxies: active — galaxies: jets — X-rays: binaries — radio continuum: galaxies — radio continuum: stars

\section{Overview}

The term 'microquasar' was proposed to designate faint Seyfert galaxies and LINERs (Netzer 1990) but is now commonly used to describe Galactic X-ray binaries which show relativistic outflows/jets (see Mirabel \& Rodríguez 1998, 1999; Fender 2001, 2002 for reviews of Galactic jet sources). Galactic microquasars (hereafter microquasars) are X-ray binaries containing an accreting black hole or a neutron star. They are generally regarded as the counterparts of more powerful extragalactic sources, such as radio-loud quasars and blazars. Although the family of relativistic Galactic radio-jet sources includes both black-hole (e.g. GRS 1915+105: Mirabel \& Rodríguez 1994; GRO J1655-40: Tingay et al. 1995) and neutronstar X-ray binaries (e.g. SS 433: Spencer 1979, Margon 1984; Cir X-1: Fender et al. 1998; Sco X-1: Fomalont, Geldzahler, \& Bradshaw 2001), here we consider a more restrictive view and compare only Galactic black-hole $\mathrm{X}$-ray binaries with compact extragalactic radio sources.

The three common ingredients of microquasars and compact extragalactic sources are the central black hole, accretion disk, and relativistic jets - the only difference is that microquasars have a stellar-mass black hole, whereas in active galactic nuclei (AGN) it is a supermassive black hole $\left(\sim 10^{6}-10^{9} \mathrm{M}_{\odot}\right)$. The similarity in the configurations of these two types of systems (see Figure 1) leads us to believe that, in spite of their different scales, the underlying physical processes in microquasars and radio-loud AGN are similar (Mirabel \& Rodríguez 1998) and are thus manifested in their radiative properties accordingly.

Both microquasars and AGN are accretion powered, and produce strong X-ray emission. The X-ray spectra

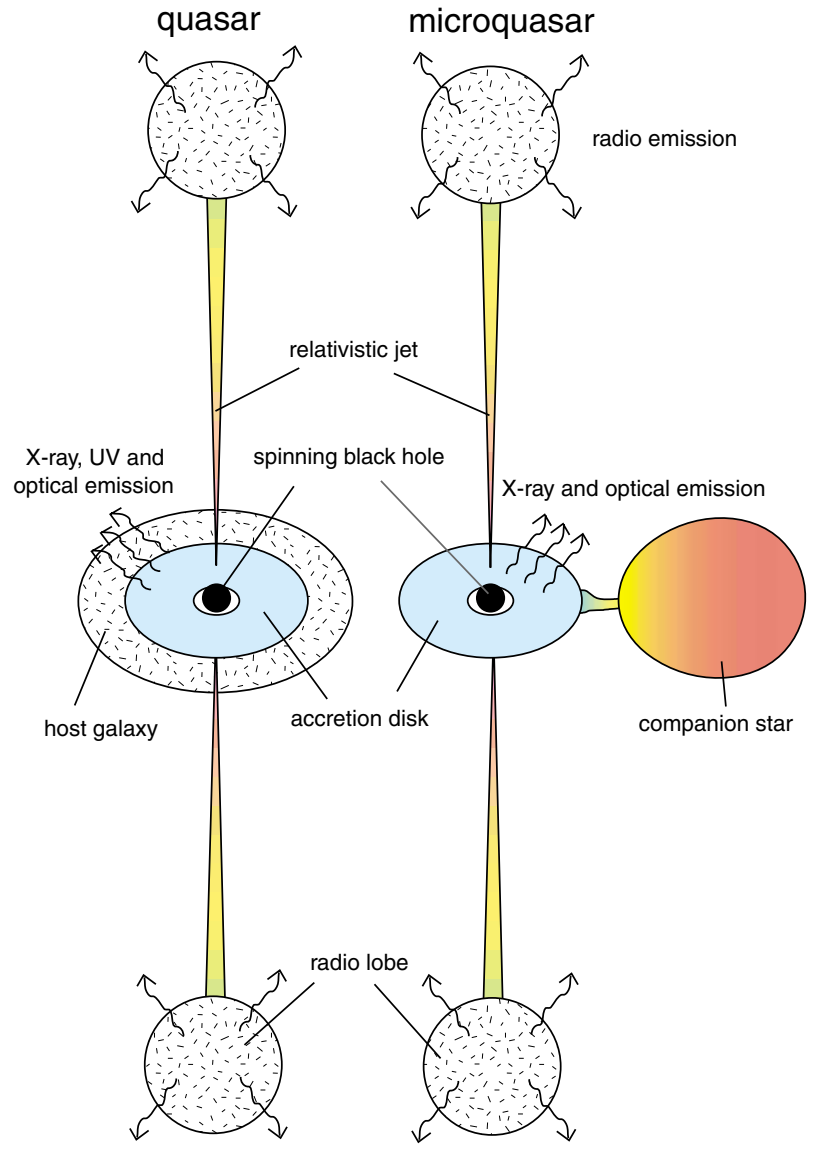

Figure 1 A schematic illustration showing the similarity between microquasars and AGN/quasars (adapted from Mirabel \& Rodríguez 1998). 
of microquasars in the soft state have a thermal blackbody component (with temperatures $\sim 1 \mathrm{keV}$ ), believed to be associated with emission from the inner region of an accretion disk, and a power-law tail (with a photon index $\Gamma \gtrsim 2$ ), that is generally interpreted as Comptonised emission arising from hot thermal $(k T \gg 1 \mathrm{keV})$ or high-speed electrons $(v \sim c)$ very close to the black hole (Sunyaev \& Titarchuk 1980; Titarchuk 1994). There are, however, reports that Galactic black-hole binaries, e.g. Cyg X-1 (Moskalenko, Collmar, \& Schönfelder 1998; McConnell et al. 2000), have a power-law spectrum extending to $\mathrm{MeV}$ energies without a cutoff. It is suggested that the highenergy tail of the extended power-law spectrum is caused by a temperature gradient in the emission region (e.g. Skibo \& Dermer 1995) and/or the presence of populations of high-energy non-thermal electrons (e.g. Gierlinski et al. 1999). Whether or not there is an extended powerlaw tail is still controversial. In the hard state the spectra are dominated by a power law, with a slope $(\Gamma \sim 1.5)$ flatter than that of the power law in the soft state. The power law in the hard state is often explained by Comptonisation models (e.g. Chakrabarti \& Titarchuk 1995; Dove, Wilms, \& Begelman 1997; Gierlinski et al. 1997; Wilms et al. 1999). Recently an alternative model was proposed, in which it is the high-energy tail of a broad synchrotron continuum emitted from relativistic jets (Markoff, Falcke, $\&$ Fender 2001). Black-body (Córdova et al. 1992) and power-law components (Mushotzky 1984; Nandra \& Pounds 1994) are also found in the UV/X-ray spectra of AGN. The black-body and power-law components in AGN also originate from accreting/outflow matter near the central engine, as in the case of Galactic black-hole systems. The black-body components in AGN spectra have lower temperatures $(\sim 0.1 \mathrm{keV})$ because of more massive (larger) black holes and hence larger inner radii for the accretion disks.

In the optical wavebands, the connection between microquasars and radio-loud AGN is less certain. While the optical emission from X-ray binaries arises in the accretion disk and from the companion star, in AGN the accretion disk, irradiatively heated clouds, supernova shocks, and stars may all contribute. During their X-ray active states, microquasars show a variety of broad and narrow emission lines. The lines can be single-peaked, double-peaked, or flat-topped depending on the excitation and ionisation states of the elements, and line morphology changes with the X-ray hardness and luminosity (Soria, Wu, \& Hunstead 2000; Wu et al. 2001). The profiles of the broad lines (e.g. the flat-topped broad lines in the spectrum of GRO J1655-40) do show some degree of resemblance to those of AGN. In both cases, these broad lines are probably emitted from high-speed matter at some radii from the central X-ray sources, where accretion and wind/outflow operate simultaneously (Murray \& Chiang 1997; Soria et al. 2000).

At radio frequencies, the main argument for unifying microquasars and extragalactic radio sources is at present based on the detection of relativistic outflows and the synchrotron origin of the radio emission. Further support is provided by the discovery of superluminal ejections from a number of sources (GRS 1915+105: Mirabel \& Rodríguez 1994; GRO J1655-40: Tingay et al. 1995; Hjellming \& Rupen 1995; XTE J1550-564: Hannikainen et al. 2001a,b). Here, we review briefly the radio properties of microquasars, with an emphasis given to comparison with extragalactic radio sources. The microquasar GRO J1655-40 is used as an illustrative example to show that the temporal-spectral properties of microquasars can be explained in terms of the generalised-shock model (Marscher \& Gear 1985) originally proposed for compact extragalactic radio sources.

\section{Radio Properties of Compact Extragalactic Sources}

Compact extragalactic radio sources often show powerlaw spectra at high frequencies $(>90 \mathrm{GHz}$; e.g. Gear et al. 1994). Coupled with high levels of linear polarisation this result provides strong evidence for optically thin synchrotron emission from non-thermal relativistic electrons. The spectra of many sources have a turnover at low frequencies, which is generally attributed to optical depth effects: the radiation is self-absorbed and becomes opaque below a critical frequency. The time evolution of the lowfrequency (less than a few $\mathrm{GHz}$ ) radio spectra was initially explained qualitatively by the adiabatic expanding-cloud model (sometimes also known as the synchrotron-bubble model, see Section 4) and its variations (Shklovsky 1965; van der Laan 1966; Pauliny-Toth \& Kellermann 1966). However, these models fail quantitatively to match the observed behaviour at low radio frequencies. Specifically, they predict a more rapid decline of peak flare amplitude, and longer time delays between high and low frequencies than is actually observed (e.g. Altschuler \& Wardle 1977). Once radio observations were made at higher frequencies (say in the sub-mm wavelengths) it became obvious that the synchrotron-bubble models failed completely to reproduce even the qualitative behaviour of the sources (e.g. Robson et al. 1983; Aller et al. 1985; see the relative time delays of flares of 3C 273 at different frequencies shown in Figure 2). These observations led to a new genre of theoretical models invoking shocks that accelerate electrons to relativistic energies as they propagate along a relativistic jet (Marscher \& Gear 1985; Hughes, Aller, \& Aller 1985). Subsequent multi-frequency datasets have shown good (though not perfect) agreement with these models (e.g. Valtaoja et al. 1988; Stevens et al. 1998; Türler, Courvoisier, \& Paltani 1999).

\section{Radio Emission from Microquasars}

To date radio emission is detected in about $30 \mathrm{X}$-ray binaries (R. P. Fender, private communication). More than 10 sources have shown resolved structure in their radio maps, indicating the presence of large-scale jets/outflows (Table 1). These jet sources are an inhomogeneous group 

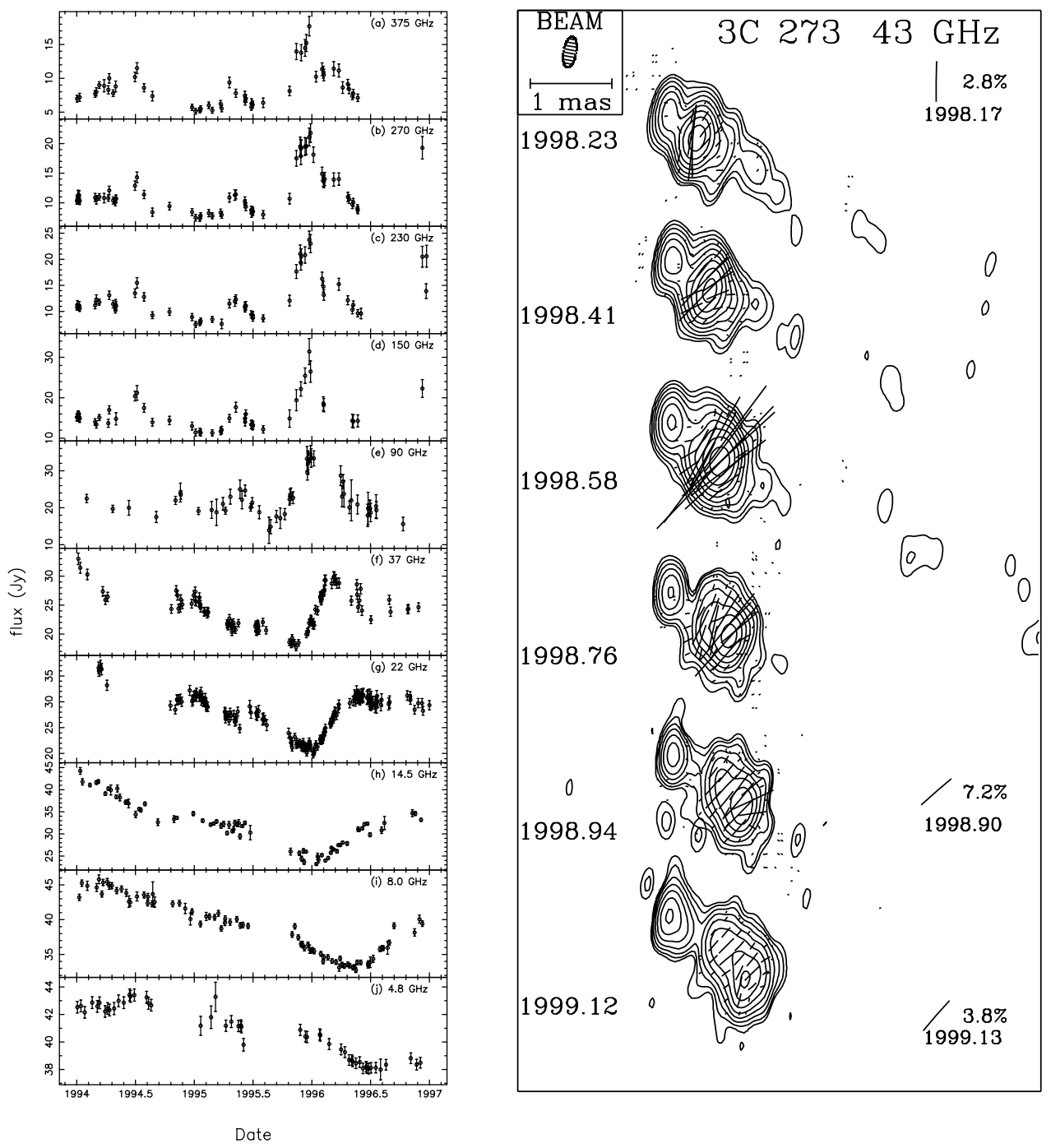

Figure 2 Left panel: The 4.8-375 GHz radio light curves of the quasar 3C 273 from 1994 to 1997 (Stevens et al. 1998). Note the prominent flare in the high frequency during late 1995 and its subsequent evolution to lower frequencies. Right panel: Bimonthly $43 \mathrm{GHz}$ VLBI polarisation image of 3C 273 taken at the VLBA (S. G. Jorstad et al., in preparation). The sticks on the images represent the electric vector position angle whilst those to the right indicate higher frequency single-dish measurements made at the James Clerk Maxwell Telescope. There is clear evidence of source expansion (cf. Figure 3).

Table 1. A list of Galactic relativistic-jet sources

The abbreviations for the references can be found in the reference list

\begin{tabular}{lllll}
\hline Source & Compact object & $V_{\text {app }}$ & \multicolumn{1}{c}{$V_{\text {int }}$} & References \\
\hline 1E 1740.7-2942 & Black hole & & $0.4-0.7 \mathrm{c}$ & Mi92, MR99 \\
GRS 1758-258 & Black hole & & & MR99 \\
GRS 1915+105 & Black hole & $1.2-1.7 \mathrm{c}$ & $0.92-0.98 \mathrm{c}$ & MR94, Fe99 \\
GRO J1655-40 & Black hole & $1.1 \mathrm{c}$ & $0.92 \mathrm{c}$ & Ti95, HR95 \\
XTE J1550-564 & Black hole & $>2 \mathrm{c}$ & & Ha01a,b \\
XTE J1748-288 & Black hole & $1.3 \mathrm{c}$ & $>0.9 \mathrm{c}$ & Hj98b \\
SAX J1819.3-2525 & Black hole & $1.0-3.2 \mathrm{c}$ & $0.85 \mathrm{c}$ & Hj00 \\
Cyg X-1 & Black hole & & $>0.6 \mathrm{c}$ & St01 \\
LS 5039 & & $\sim 0.4 \mathrm{c}$ & Pa00 \\
SS 433 & Neutron star? & $0.26 \mathrm{c}$ & $0.26 \mathrm{c}$ & Ma84, Sp84 \\
Cyg X-3 & Neutron star? & & $\geq 0.8 \mathrm{c}$ & Sc95, MP01, MR01 \\
CI Cam & Neutron star? & $\sim 0.15 \mathrm{c}$ & $\sim 0.15 \mathrm{c}$ & Hj98a \\
Sco X-1 & Neutron star & $\sim 0.5 \mathrm{c}$ & & FG01 \\
Cir X-1 & Neutron star & $\geq 0.1 \mathrm{c}$ & $\geq 0.1 \mathrm{c}$ & St93, Fe98 \\
\hline
\end{tabular}


of objects. Some are persistent sources with no significant variations in the radio flux densities over timescales longer than half a decade (e.g. 1E 1740.7-2942 and GRS 1758-258, Mirabel \& Rodríguez 1999). A significant proportion are transients with radio flaring activity only in the X-ray active states. Some of the transient sources also show superluminal ejection events (e.g. GRS 1915+105, see Figure 3).

The temporal and spectral properties of the radio emission from the (transient) microquasars are not all identical. This can be seen in the light curves of four systems, GRO J1655-40, GRS 1915+105, XTE J1550-564, and GX 339-4 observed by the Molongo Observatory Synthesis Telescope (MOST) of the University of Sydney (Figure 4). The radio outbursts of microquasars are found to have certain associations with the X-ray variations (Mirabel \& Rodríguez 1999; Fender 2002), but the association may vary from system to system. For example, simultaneous quenching of radio emission and hard (>20 keV) X-rays was observed in GX339-4 (Fender et al. 1999b), while for XTE J1550-564 a largeamplitude radio flare occurred almost simultaneously with the X-ray flare during its 1998 outburst (Hannikainen et al. 2001b; Wu et al. 2002).

In GRS 1915+105, there is some correlation during the so-called short-term ( $\sim 20-30$ days) 'lulls' when both the soft X-rays and the radio emission drop to levels of low, steady emission, but the hard X-rays do not seem to vary in accordance. (The lulls usually occur in the midst of chaotic flaring activity in both the radio and soft X-rays, see e.g. Hannikainen 1999.) In GRO J1655-40, the maxima of the radio flares in 1994 occurred with some time delay (approximately 5-14 days) with respect to the peaks of the hard X-ray bursts (Hannikainen et al. 2000). However, the following period of hard X-ray activity, which occurred in 1995, showed no accompanying radio emission (Tavani et al. 1996).

The radio emission from microquasars is linearly polarised, indicating its synchrotron origin (Fender et al. 1999a; Hannikainen et al. 2000). During the ejection events of GRO J1655-40, the linear polarisation reached levels of up to $\sim 12 \%$ at high frequencies $(8.6$ and $9.2 \mathrm{GHz}$ ) and $\sim 8-10 \%$ at lower frequencies $(1.4-4.8 \mathrm{GHz})$. Circular polarisation was also detected in GRO J1655-40 during the 1994 outburst (Macquart et al. 2002; circular polarisation is detected in another jet source SS 433, Fender et al. 2000).

\section{Bubble Model vs Shock Model}

\subsection{Synchrotron-bubble Model}

The spectral evolution of microquasars has been explained by the synchrotron-bubble model (Hjellming \& Johnston 1988; Ball \& Vlassis 1993; Hjellming \& Han 1995). The model assumes that the radio emission is emitted from a cloud composed of relativistic electrons and a randomly oriented magnetic field, and that the energy densities of the relativistic electrons and the magnetic field are in

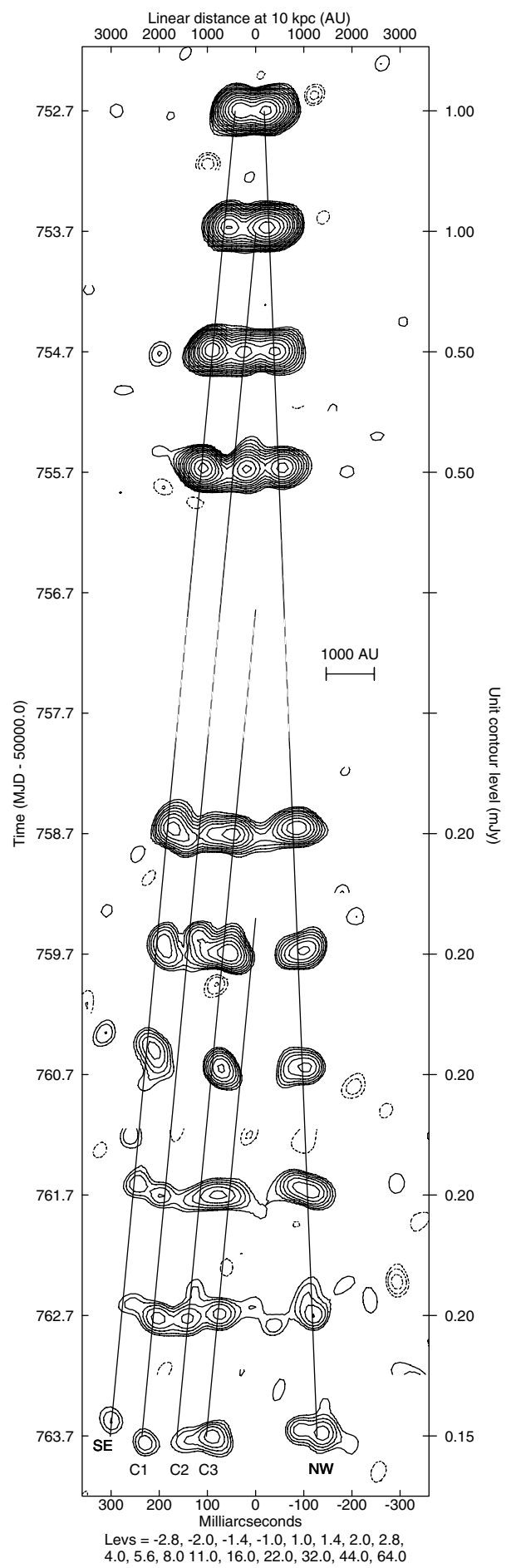

Figure 3 The total intensity MERLIN image of GRS $1915+105$ (from Fender et al. 1999a). Contour levels increase in factors of $\sqrt{2}$ from the unit contour level indicated at the right-hand side of each image.

equipartition. The radiative process is relatively inefficient (i.e. $t_{\text {rad }} \ll t_{\text {exp }}$, where $t_{\text {rad }}$ and $t_{\exp }$ are the timescales for radiative loss and expansion respectively), such that the electrons and magnetic field lose energy due to the (quasi-)adiabatic expansion of the cloud. The model predicts that the emission at high frequencies peaks before the emission at low frequencies (Figure 5, left panel). Also, the 

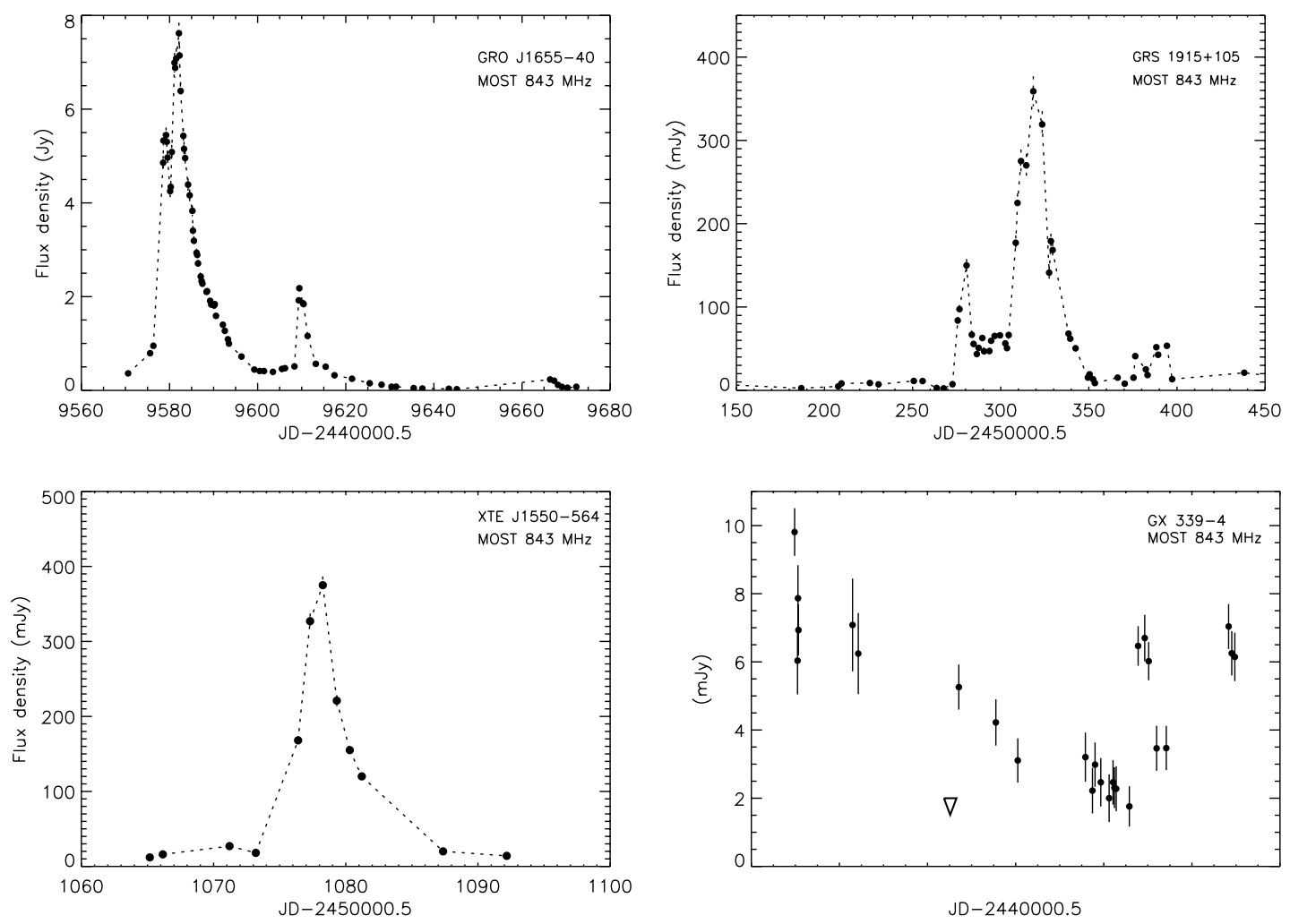

Figure 4 The $843 \mathrm{MHz}$ radio light curves of four candidate black-hole binaries, GRO J1655-40 (top left), GRS 1915+105 (top right), XTE J1550-564 (bottom left), and GX 339-4 (bottom right), observed by the MOST of Sydney University.
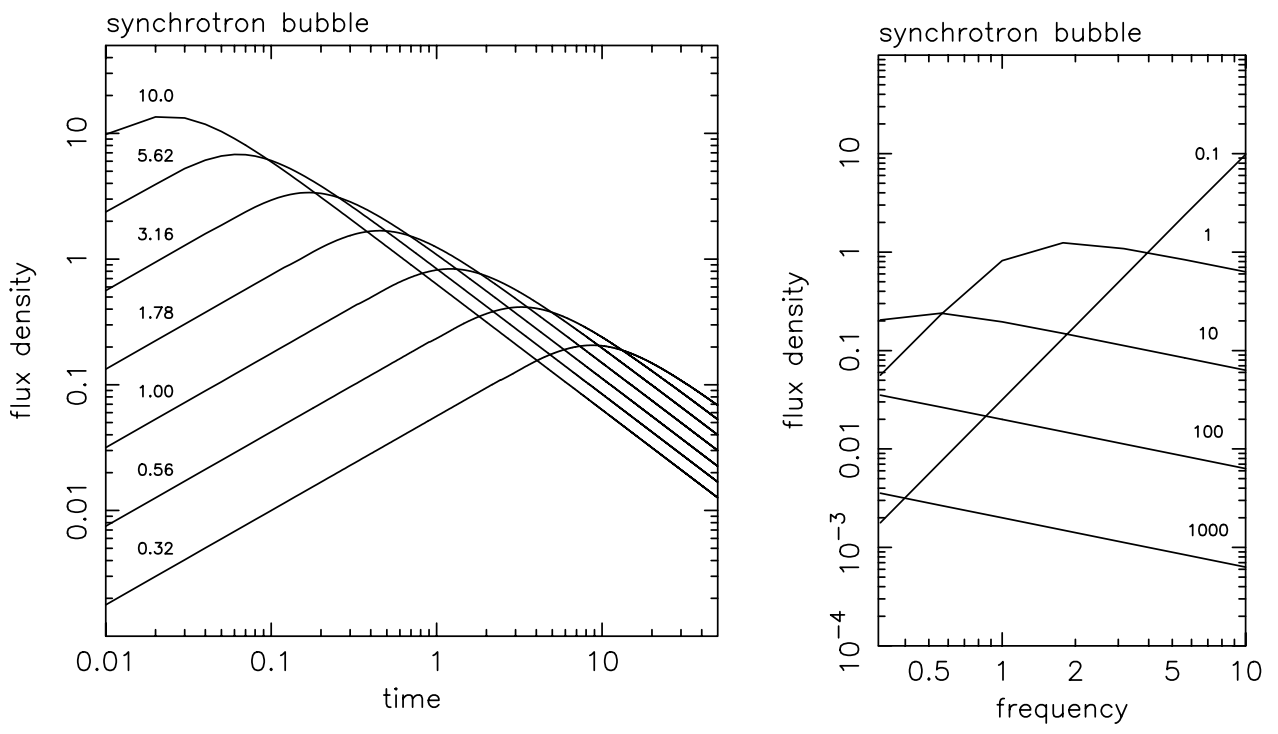

Figure 5 The multi-frequency light curves and spectra (left and right panel respectively) of the synchrotron-bubble model. The flux densities $S_{v}$ are calculated according to the prescription given in Hjellming \& Johnston (1988) and Hjellming \& Han (1995). The radio emission region is assumed to be spherical and the relativistic electrons have an energy distribution $N(E) \propto E^{-s}$, where $s=2$. The normalisation is chosen such that $S_{v}=1$ for the frequency $v=1$ and at the epoch $t=1$. The frequencies of the light curves are $0.32,0.56,1.00,1.78,3.16,5.62$, and 10.0 (in arbitrary units). The time of the spectra are $0.1,1,10,100$, and 1000 (in arbitrary units).

peak flux density is higher for higher frequencies. Moreover, the spectrum is inverted and self-absorbed initially, and then becomes optically thin and appears as a power law as the bubble expands (Figure 5, right panel).

\subsection{Generalised-shock Model}

This model consists of an adiabatic relativistic jet in which transverse shock waves form in response to changes in pressure or bulk flow (e.g. Blandford \& Königl 1979; 


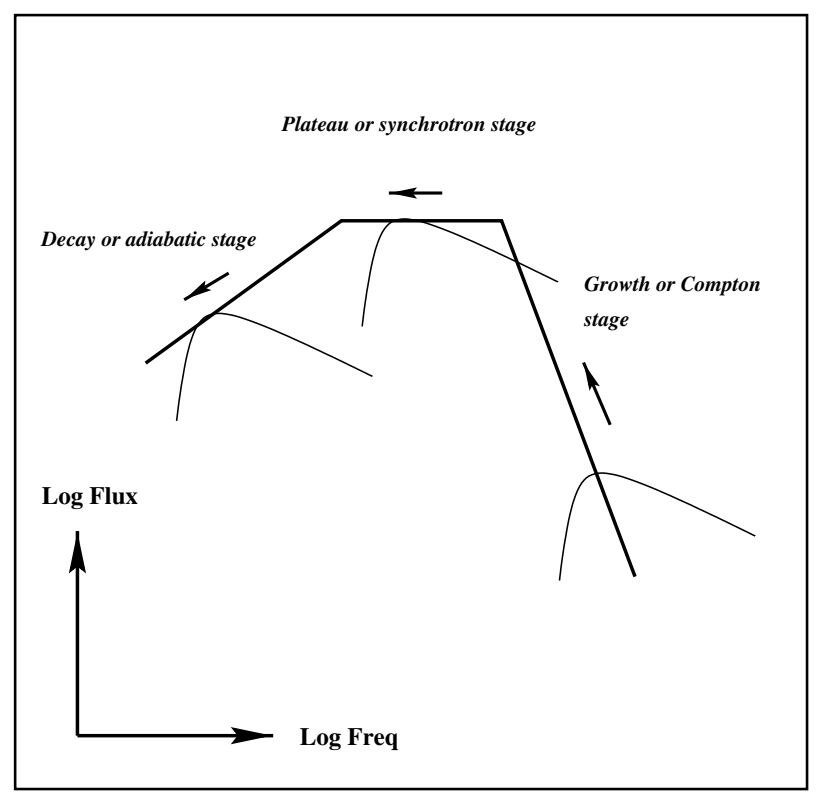

Figure 6 Schematic representation of the generalised-shock model proposed by Marscher \& Gear (1985). The development of the shock can be characterised by the growth, plateau, and decay stages.

Marscher \& Gear 1985; see also Kaiser, Sunyaev, \& Spruit 2000). The evolution of the shock is described in terms of the flux density at the peak frequency $\left[S_{v}\left(v_{\mathrm{m}}\right)=S_{\mathrm{m}}\right]$ of the synchrotron spectrum where the opacity is close to unity. This point is determined at any one time by the dominant energy-loss mechanism. When the emitting region is compact, inverse-Compton losses predominate (Compton or growth stage) but these fall off rapidly as the shock expands and are superseded by synchrotron losses (synchrotron or plateau stage). As the shock expands further, the radiative lifetime of the electrons becomes long with respect to the time needed to traverse the emitting region and losses due to adiabatic expansion (adiabatic or decay stage) become more important. These model predictions for the spectral evolution during flaring events are shown schematically in Figure 6 . The spectra at all three stages are approximated by power laws on the logarithmic $\left(S_{\mathrm{m}}, v_{\mathrm{m}}\right)$ plane. As the emitting region expands, $v_{\mathrm{m}}$ is predicted to move to lower frequencies with time. $S_{\mathrm{m}}$ increases rapidly during the Compton stage, remains approximately constant during the synchrotron stage and decreases during the adiabatic stage.

The maximum flare amplitude for monitoring frequencies on the growth stage occurs when the spectrum transits onto the plateau stage. Light curves at monitoring frequencies on the growth stage are thus predicted to peak simultaneously, and because of the spectral shape, the flare amplitudes are expected to increase towards lower frequency - specifically, they should have the same power-law form as the optically thin portion of the flare spectrum. Flare amplitudes at monitoring frequencies commensurate with the later stages are determined by the details of the spectral evolution, and are thus expected to be approximately constant during the plateau stage and to decrease during the decay stage. The light curves will display time-lagged behaviour with a delay between any two frequencies equal to the time taken for the spectrum to evolve between them and become optically thin. (For a detailed discussion on the application of the model to the multi-frequency light curves of extragalactic radio sources, see e.g. Valtaoja et al. (1992).)

\section{The 1994 Radio Outburst of GRO J1655-40}

To date GRO J1655-40 and GRS 1915+105 are the two Galactic sources that have shown unambiguous superluminal ejecta. GRS $1915+105$ is a black-hole binary with a low mass K-M III giant star (Greiner et al. 2001), and GRO J1655-40 contains an evolved F-type star (Orosz \& Bailyn 1997; Soria et al. 1998).

In 1994 GRO J1655-40 showed a dramatic radio outburst: the flux density at $843 \mathrm{MHz}$ increased from a few hundred mJy to almost $8 \mathrm{Jy}$ in about 13 days (Hannikainen et al. 2000). VLBA images taken during the outburst show collimated, relativistic jets. The jets can be resolved into several knot-like features which expand outwards from a stationary compact core (Hjellming \& Rupen 1995). The complex flaring behaviour can be seen in the multi-frequency light curves shown in Figure 7 (left panel). There are several minor events following the initial increase in flux. The key features are that the initial rise in flux peaks simultaneously at all frequencies and that the amplitude of the flare, which is maximum minus minimum flux, increases towards lower frequency.

The spectrum can be described as a power law most of the time, but it becomes inverted when flaring begins (Hannikainen et al. 2000). The spectrum just before flux maximum (Figure 7, top of the right panel) is consistent with a synchrotron spectrum which is optically thin at the highest frequencies but becomes self-absorbed at around $1 \mathrm{GHz}$. As flaring proceeds, the entire spectrum becomes optically thin and remains so throughout the subsequent decline in flux density. From the two-point spectral indices, we can see that the spectrum flattens as the flux initially declines. It then steepens again around the time of the second outburst. A possible explanation is that the emission arises from several regions. Hannikainen et al. (2000) proposed a phenomenological model in which steep spectrum flare emission from more extended lobes is superposed onto flatter emission from a compact core. The spectral variations are the consequences of variations in the relative brightness of the lobes and the core. The exact nature of the emitting lobes and core is, however, not specified.

The time-dependent spectral properties of the 1994 outburst of GRO J1655-40 contradict the prediction of the synchrotron-bubble model for radio emission from transient X-ray binaries but can in fact be easily explained by the generalised-shock model. The simultaneous peaking of the light curves at all radio frequencies is consistent with the properties of the Compton/growth stage if the transition to the synchrotron/plateau stage is at a frequency lower than the frequencies of the observation 

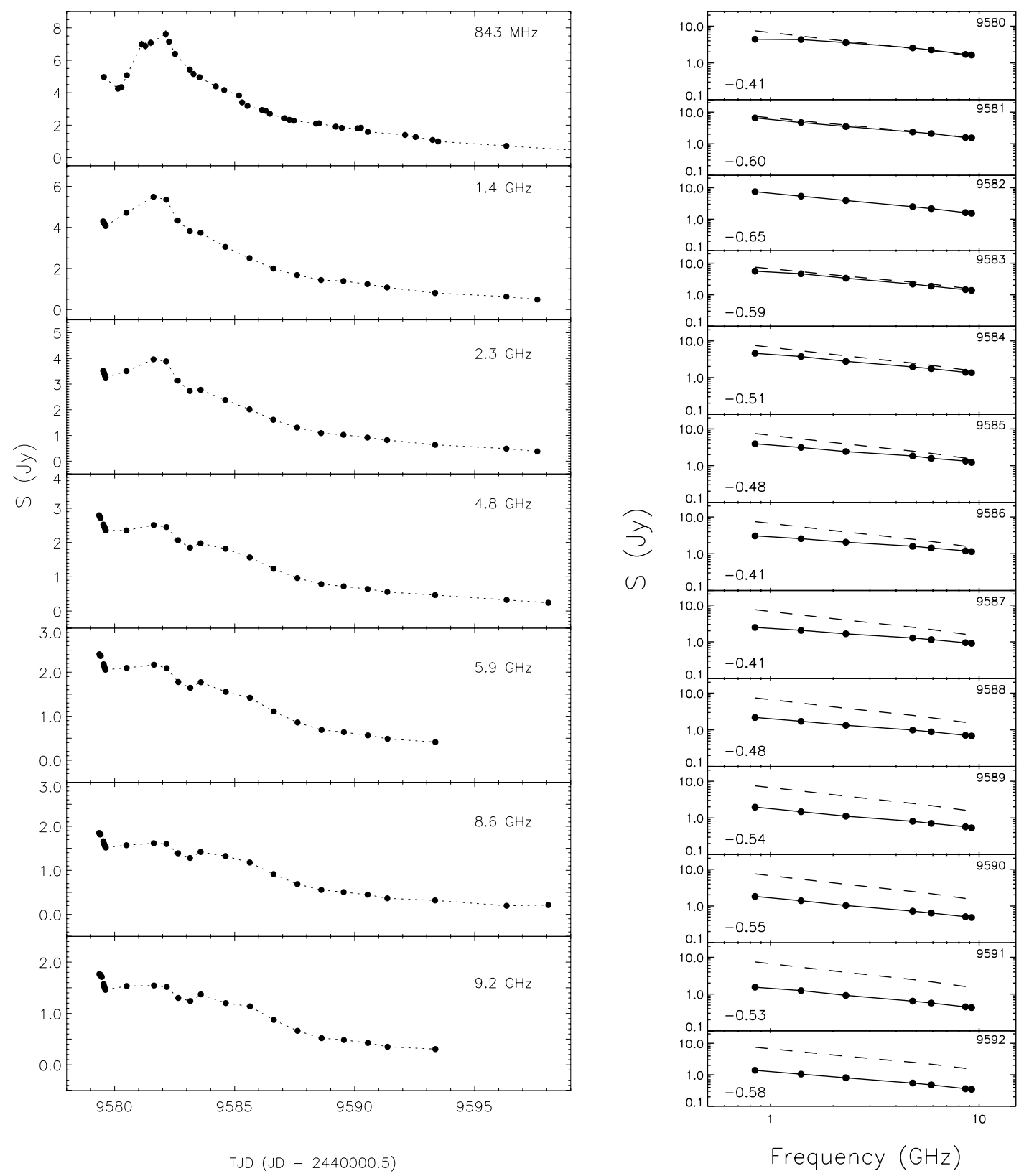

Figure 7 The multi-frequency radio light curves and spectra of GRO J1655-40 during the 1994 outbursts (left and right panel respectively, from Stevens et al. 2002). The dashed lines in the right panel are the spectra at TJD 9582 (shown as a reference to illustrate the spectral evolution). The two-point (highest and lowest frequencies) spectral indices are shown in the lower corner of each sub-panel.

(Figure 6). The flare amplitudes fall off as a power law at the Compton/growth stage, and they have the same frequency dependence until it reaches the transition point between the growth and plateau stages. The flare amplitudes of GRO $\mathrm{J} 1655-40$, normalised to $4.8 \mathrm{GHz}$ is a power law with a slope of -0.7 (Figure 8 ), similar to the optically thin portion of the flare synchrotron spectrum of compact extragalactic radio sources.

One apparent difference between the flaring behaviour of GRO J1655-40 and its extragalactic analogues, the blazars, is that the flare remains in the growth stage to much lower frequencies. For example, many of the sources in the sample considered in Stevens et al. (1994) show delayed emission in the frequency range $37-90 \mathrm{GHz}$, although some BL Lacertae objects remained in the growth stage down to $4.8 \mathrm{GHz}$. A contributor to this effect is the Doppler factor which will shift the emitted radiation towards higher observed frequencies for the blazars but will have little (or possibly slightly the opposite) effect for the jets of GRO J1655-40 (which has an orbital inclination of $\approx 70^{\circ}$, Orosz \& Bailyn 1997). Another contributing factor is probably the physical differences between jets of Galactic and extragalactic sources. The flare evolution depends on the photon energy density, the magnetic energy density, and the lifetime of the emitting electrons compared to the time it takes them to cross the shock structure, and hence it is dependent on the size of the emitting region and its magnetic-field strength. 


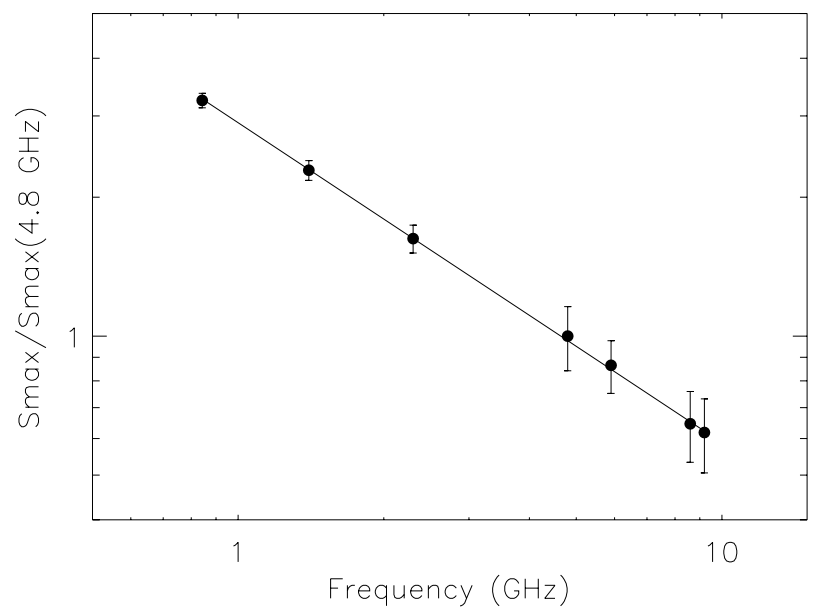

Figure 8 Flare amplitudes for the 1994 outburst of GRO J1655-40 normalised to $4.8 \mathrm{GHz}$ (from Stevens et al. 2002). The slope of the fit is $-0.69 \pm 0.05$.

The multi-frequency light curves presented in Hjellming \& Rupen (1995), which include data up to $22.5 \mathrm{GHz}$, show that the decay times are frequency dependent as expected for synchrotron losses (but not for expansion losses which are independent of frequency, see Figure 5). For synchrotron losses, the magnetic field, $B$, can be estimated from $B \sim 68\left(v_{\mathrm{m}} / 1 \mathrm{GHz}\right)^{-1 / 3} \times$ $(t / 1 \mathrm{~d})^{-2 / 3} \delta^{-1 / 3}$ where $\nu_{\mathrm{m}}$ is the observing peak frequency and $t$ is the e-folding decay time. (The relativistic Doppler factor, $\delta=\Gamma^{-1}(1-\beta \cos \theta)^{-1}$, where $\Gamma=\left(1-\beta^{2}\right)^{-1 / 2}$ is the bulk Lorentz factor, $\theta$ is the viewing angle, and $\beta$ is the jet speed in units of $c$.) For $\theta=70^{\circ}$, $\beta=0.9$ gives $\delta \sim 1.0$. The flare decay time estimated from the $843 \mathrm{MHz}$ light curve is 5 days, implying a magnetic field of $\sim 25$ Gauss (cf. the typical fields of $\sim 0.1-1$ Gauss for extragalactic sources, e.g. Brown et al. 1989).

The radio data of GRO J1655-40 have clearly shown that generalised-shock models (e.g. Marscher \& Gear 1985; Kaiser et al. 2000) are more consistent with the outburst behaviour of microquasars than the conventional synchrotron-bubble models (e.g. Hjellming \& Johnston 1988). It is also worth noting that the RXTE data of GRS 1915+105 also point toward the shock models (see Yadav 2001). A more detailed quantitative discussion of the radio properties of microquasars in terms of the generalised-shock model can be found in Stevens et al. (2002).

\section{Summary}

There is strong evidence that the physical processes that give rise to radio emission from microquasars and compact extragalactic radio sources are similar. In particular, both can be explained by the generalised-shock models, in which shocks are propagating in the relativistic jets, and synchrotron radiation is emitted from the shockaccelerated relativistic electrons. The spectral evolution of the microquasar GRO J1655-40 in the radio waveband during the 1994 outburst is consistent with the predictions of the generalised-shock models, and the data suggest that the spectral evolution of the system was in the growth phase. The electrons lose energy mainly via radiative processes, and not via adiabatic expansion of the electron cloud as in conventional synchrotron-bubble models.

\section{Acknowledgments}

We thank R. Hunstead, R. Fender, and J.-P. Macquart for discussions, and R. Fender for the MERLIN image of GRS 1915+105. We also thank an anonymous referee for helpful comments on the X-ray spectral properties of Galactic black-hole binaries and suggestions on improving the manuscript. DCH acknowledges a PPARC grant to the University of Southampton and the travel support from the Academy of Finland. KW acknowledges the support from the ARC through an Australian Research Fellowship. This work is partially supported by a University of Sydney Sesqui R\&D Grant.

\section{References}

Aller, H. D., Aller, M. F., Latimer, G. E., \& Hodge, P. E. 1985, ApJS, 59,513

Altschuler, D. R., \& Wardle, J. F. C. 1977, MNRAS, 179, 153

Ball, L., \& Vlassis, M. 1993, PASA, 10, 342

Blandford, R. D., \& Königl, A. 1979, ApJ, 232, 34

Brown, L. M. J., et al. 1989, ApJ, 340, 129

Chakrabarti, S., \& Titarchuk, L. G. 1995, ApJ, 455, 623

Córdova, F. A., Kartje, J. F., Thompson, R. J., Mason, K. O., Puchnarewicz, E. M., \& Harnden, F. R. 1992, ApJS, 81, 661

Dove, J. B., Wilms, J., \& Begelman, M. C. 1997, ApJ, 487, 759

Fender, R. P. 2001, APSSS, 276, 69

Fender, R. P. 2002, in Relativistic Outflows in Astrophysics, Springer Lecture Notes in Physics, eds A. W. Guthman, M. Georganopoulous, \& A. Macrowith (Berlin: SpringerVerlag), in press

Fender, R. P., Rayner, D., Norris, R., Sault, R. J., \& Pooley, G. 2000, ApJ, L29

Fender, R. P., Spencer, R., Tzioumis, T., Wu, K., van der Klis, M., van Paradijs, J., \& Johnston, H. 1998, ApJ, 506, L121 (Fe98)

Fender, R. P., Garrington, S. T., McKay, D. J., Muxlow, T. W. B., Pooley, G. G., Spencer, R. E., Stirling, A. M., \& Waltman, E. B. 1999a, MNRAS, 304, 865 (Fe99)

Fender, R. P., et al. 1999b, ApJ, 519, L165

Fomalont, E. B., Geldzahler, B. J., \& Bradshaw, C. F. 2001, ApJ, 553, L27 (FG01)

Gear, W. K., et al. 1994, MNRAS, 267, 167

Gierlinski, M., Zdziarski, A. A., Done, C., Johnson, W. N., Ebisawa, K., Ueda, Y., Haardt, F., \& Phlips, B. 1997, MNRAS, 288, 958

Gierlinski, M., Zdziarski, A. A., Poutanen, J., Coppi, P. S., Ebisawa, K., \& Johnson, W. N. 1999, MNRAS, 309, 496

Greiner, J., Cuby, J. G., McCaughrean, M. J., Castro-Tirado, A. J., \& Mennickent, R. E. 2001, A\&A, 373, L37

Hannikainen, D. C. 1999, PhD Thesis, University of Helsinki

Hannikainen, D. C., Hunstead, R. W., Campbell-Wilson, D., Wu, K., McKay, D. J., Smits, D. P., \& Sault, R. J. 2000, ApJ, 540, 521

Hannikainen, D., Campbell-Wilson, D., Hunstead, R. W., McIntyre, V., Lovell, J., Reynolds, J., Tzioumis, T., \& Wu, K. 2001a, APSS, 276, 45 (Ha01a)

Hannikainen, D., et al. 2001b, in Exploring the Gamma-Ray Universe: Proceedings of the 4th INTEGRAL Workshop, eds A. Gimenez, V. Reglero, \& C. Winkler, ESA SP-459, 291 (Ha01b) 
Hjellming, R. M., \& Han, X. 1995, in X-ray Binaries, eds W. H. G. Lewin, J. van Paradijs, \& E. P. J. van den Heuvel (Cambridge: Cambridge University Press), 308

Hjellming, R. M., \& Johnston, K. J. 1988, ApJ, 328, 600

Hjellming, R. M., \& Rupen, M. P. 1995, Nature, 375, 464 (HR95)

Hjellming, R. M., Mioduszewski, A. J., Ghigo, F. D., Rupen, M. P., \& Waltman, E. B. 1998, BAAS, 192, 78.05 (Hj98a)

Hjellming, R. M., Rupen, M. P., Mioduszewski, A. J., Smith, D. A., Harmon, B. A., Waltman, E. B., Ghigo, F. D., \& Pooley, G. G. 1998, BAAS, 193, 10.398 (Hj98b)

Hjellming, R. M., et al. 2000, ApJ, 544, 977 (Hj00)

Hughes, P. A., Aller, H. D., \& Aller, M. F. 1985, ApJ, 298, 310

Kaiser, C. R., Sunyaev, R., \& Spruit, H. C. 2000, A\&A, 356, 975

Macquart, J.-P., Wu, K., Sault, R., \& Hannikainen, D. C. 2002, A\&A, submitted

Margon, B. A. 1984, ARA\&A, 22, 507 (Ma84)

Markoff, S., Falcke, H., \& Fender, R. 2001, A\&A, 372, L25

Marscher, A. P., \& Gear, W. K. 1985, ApJ, 298, 114

Martí, J., Paredes, J. M., \& Peracaula, M. 2001, A\&A, 375, 476 (MP01)

McConnell, M. L., et al. 2000, ApJ, 543, 928

Mioduszewski, A. M., Rupen, M. R., Hjellming, R. M., Pooley, G. G., \& Waltman, E. B. 2001, ApJ, 553, 766 (MR01)

Mirabel, I. F., \& Rodríguez, L. F. 1994, Nature, 371, 46 (MR94)

Mirabel, I. F., \& Rodríguez, L. F. 1998, Nature, 392, 673

Mirabel, I. F., \& Rodríguez, L. F. 1999, ARA\&A, 37, 409 (MR99)

Mirabel, I. F., Rodríguez, L. F., Cordier, B., Paul, J., \& Lebrun, F. 1992, Nature, 358, 215 (Mi92)

Moskalenko, I. V., Collmar, W., \& Schönfelder, V. 1998, ApJ, 502,428

Murray, N., \& Chiang, J. 1997, ApJ, 474, 91

Mushotzky, R. F. 1984, AdSpR, 3, 157

Nandra, K., \& Pounds, K. A. 1994, MNRAS, 268, 405

Netzer, H. 1990, in Active Galactic Nuclei, Saas-Fee Advance Course 20 Lecture Notes, eds T. J. L. Courvoisier, \& M. Mayor (Berlin: Springer-Verlag), 57

Orosz, J. A., \& Bailyn, C. D. 1997, ApJ, 477, 876

Paredes, J. M., Marti, J., Ribo, M., \& Massi, M. 2000, Science, 288, $2340(\mathrm{~Pa} 00)$
Pauliny-Toth, I. I. K., \& Kellermann, K. I. 1966, ApJ, 146, 634

Robson, E. I., et al. 1983, Nature, 305, 194

Schalinski, C. J., et al. 1995, ApJ, 447, 752 (Sc95)

Shklovsky, I. S. 1965, SvA, 7, 748

Skibo, J. G., \& Dermer, C. D. 1995, ApJ, 455, L25

Soria, R., Wu, K., \& Hunstead, R. W. 2000, ApJ, 539, 445

Soria, R., Wickramasinghe, D. T., Hunstead, R. W., \& Wu, K. 1998, ApJ, 495, L95

Spencer, R. E. 1979, Nature, 282, 483

Spencer, R. E. 1984, MNRAS, 209, 869 (Sp84)

Stewart, R. T., Caswell, J. L., Haynes, R. F., \& Nelson, G. J. 1993, MNRAS, 261, 593 (St93)

Stevens, J. A., Hannikainen, D. C., Wu, K., Hunstead, R. W., \& McKay, D. 2002, MNRAS, submitted

Stevens, J. A., Litchfield, S. J., Robson, E. I., Hughes, D. H., Gear, W. K., Teräsranta, H., Valtaoja, E., \& Tornikoski, M. 1994, ApJ, 437, 91

Stevens, J. A., Robson, E. I., Gear, W. K., Cawthorne, T. V., Aller, M. F., Aller, H. D., Teräsranta, H., \& Wright, M. C. H. 1998, ApJ, 502, 182

Stirling, A. M., Spencer, R. E., de la Force, C. J., Garrett, M. A., Fender, R. P., \& Ogley, R. N. 2001, MNRAS, 327, 1273 (St01)

Sunyaev, R. A., \& Titarchuk, L. G. 1980, A\&A, 86, 121

Tavani, M., Fruchter, A., Zhang, S. N., Harmon, B. A., Hjellming, R. M., Rupen, M. P., Bailyn, C., \& Livio, M. 1996, ApJ, 473, L103

Tingay, S. J., et al. 1995, Nature, 374, 141 (Ti95)

Titarchuk, L. 1994, ApJ, 434, 570

Türler, M., Courvoisier, T. J.-L., \& Paltani, S. 1999, A\&A, 349, 45

Valtaoja, E., Teräsranta, H., Urpo, S., Nesterov, N. S., Lainela, M., \& Valtonen, M. 1992, A\&A, 254, 71

Valtaoja, E., et al. 1988, A\&A, 203, 1

van der Laan, H. 1966, Nature, 211, 1131

Wilms, J., Nowak, M. A., Dove, J. B., Fender, R., \& di Matteo, T. 1999, ApJ, 522, 460

Wu, K., Soria, R., Hunstead, R. W., \& Johnston, H. M. 2001, MNRAS, 320, 177

Wu, K., et al. 2002, ApJ, 565, 1161

Yadav, J. S. 2001, ApJ, 548, 876 